\title{
Direct observation of conduction band plasmons and the related Burstein-Moss shift in highly doped semiconductors: A STEM-EELS study of Ga-doped ZnO
}

\author{
Cecilie S. Granerød, ${ }^{1, *}$ Sindre R. Bilden, ${ }^{1}$ Thomas Aarholt, ${ }^{1}$ Yu-Feng Yao, ${ }^{2}$ C. C. Yang, ${ }^{2}$ David C. Look,${ }^{3}$ Lasse Vines, ${ }^{1}$ \\ Klaus Magnus Johansen, ${ }^{1}$ and Øystein Prytz ${ }^{1}$ \\ ${ }^{1}$ Department of Physics, Centre for Materials Science and Nanotechnology, University of Oslo, P. O. Box 1048 Blindern, N-0316 Oslo, Norway \\ ${ }^{2}$ Institute of Photonics and Optoelectronics, National Taiwan University, No. 1, Section 4, Roosevelt Road, Taipei, 10617 Taiwan \\ ${ }^{3}$ Semiconductor Research Center, Wright State University, Dayton, Ohio 45432, USA
}

(Received 1 June 2018; revised manuscript received 16 August 2018; published 6 September 2018)

\begin{abstract}
The combination of high optical transparency and low electrical resistivity has made transparent conductive oxides (TCOs) a key technology in many optoelectronic applications. Furthermore, the study of TCOs yields insight into many fundamental parameters of semiconductors. For example, the high charge carrier concentration results in an apparent shift in the band gap, the so-called Burstein-Moss shift, in addition to plasmonic resonances in the near infrared regime. While both effects are related to the carrier concentration and band structure, their lateral distribution and interaction with boundary conditions such as interfaces and surfaces are difficult to assess, and a direct observation of the local distribution has remained elusive. Here we employ electron energy-loss spectroscopy in scanning transmission electron microscopy (STEM-EELS) for direct observation of spatially resolved plasmonic resonances and Burstein-Moss shift in gallium doped zinc oxide (GZO), one of the most widely used TCOs. A $25 \mathrm{~nm}$ thick GZO film with a carrier concentration of $7 \times 10^{20} \mathrm{~cm}^{-3}$ has been grown epitaxially on a nominally undoped $\mathrm{ZnO}$ film. The GZO film shows a renormalized Burstein-Moss shift of $\sim 0.5 \mathrm{eV}$, in accordance with that expected from Hall effect measurements. The plasma resonance of the conduction band electrons is located at $0.82 \mathrm{eV}$ in the bulk GZO, and with a substantial increase in intensity towards the sample surface, where a surface plasmon energy of $0.54 \mathrm{eV}$ is observed. Hence we have directly measured differences in optical properties between the two films, the local variation across the GZO film has been studied, and we are also directly observing the difference between bulk and surface properties of GZO.
\end{abstract}

DOI: 10.1103/PhysRevB.98.115301

\section{INTRODUCTION}

The tunability of charge carrier concentrations in semiconductors is a key feature in all semiconductor components. In the low resistivity limit provided by transparent conductive oxides (TCOs), the carrier concentration is sufficiently high for a substantial filling of the conduction band, with the change in the apparent band gap as stated by Burstein and Moss [1,2], and a compensating effect known as band gap renormalization leading to band gap narrowing (BGN) [3]. It also introduces plasmonic resonance wavelengths in the near infrared regime, opening for potential use in photovoltaic devices, where TCOs are already used as transparent electrodes, and in telecommunication reaching the technologically important wavelengths of 1.30 and $1.55 \mu \mathrm{m}$.

Metal-based plasmonics have received a lot of attention the past decade, where tunability of plasmonic resonance wavelength can be achieved via scaling of the metal nanoparticles [4-6]. However, the typical carrier concentrations in metals, normally around $10^{22} \mathrm{~cm}^{-3}$, are too high to support a plasmonic resonance wavelength in the visible and IR region. On the other hand, highly doped semiconductors, including TCOs, have lower concentrations and are highly suitable for both telecom and PV applications [7-10]. One well-

*cecilie.granerod@fys.uio.no established semiconductor material that can be doped heavily enough is $\mathrm{ZnO}$, where $\mathrm{Ga}$ or $\mathrm{Al}$ are important dopants [11-14] that introduce minimal lattice distortions $[15,16]$. $\mathrm{ZnO}$ has a high conductivity and transparency due to a wide optical band gap $(\sim 3.3 \mathrm{eV})$ and a high exciton binding energy (60 meV), where the optical and electronic properties can be tuned by doping.

Charge carrier concentrations and mobilities can be measured macroscopically using Hall effect based techniques [17], or locally using probe based techniques [18], while optical properties are typically performed using characterization techniques such as UV-VIS absorption and reflectance, or photoluminescence spectroscopy. To extract information about plasmonic resonance frequencies in semiconductors, reflectance measurements are typically used [19]. Optical characterization involves interaction between light and material, and the spatial resolution of these techniques is thus diffraction limited and depends on the wavelength. However, in the development of new semiconductor devices, there is a trend towards small scale systems where optical properties may even vary at the nanoscale. Hence conventional methods for optical measurements become less suitable. One solution is to use electron energy-loss spectroscopy in scanning transmission electron microscopy (STEM-EELS) for band gap measurements. Here, band gap measurements with nanometer scale spatial resolution are possible [20-25], for example, across very thin films, thus providing band gap 
information on length scales not available with other methods. Importantly, the possibilities need to be explored on relevant materials systems. In this respect, information about the local variation in apparent band gap in highly doped semiconductor films and the influence of the local band gap variation on the plasmonic resonance wavelengths needs to be established.

Here, we demonstrate direct observation of the local distribution of the band gap and plasmonic resonance frequencies using STEM-EELS on a homoepitaxially grown stack with a nominally $25 \mathrm{~nm}$ thick film of heavily Ga-doped $\mathrm{ZnO}$ (GZO) on top of undoped $\mathrm{ZnO}$. The extracted optical parameters are in excellent agreement with charge carrier concentrations found by Hall effect measurements and reflectance measurements, thereby validating the approach. Moreover, the apparent optical band gap, corresponding to a combined BursteinMoss shift and band gap renormalization of $\sim 0.5 \mathrm{eV}$, appears to be approximately uniform throughout the film thickness. On the other hand, distinct regions are found in the plasmon energy maps, which are identified as the bulk plasmon energy and the corresponding surface plasmon energy at $0.82 \mathrm{eV}$ and $0.56 \mathrm{eV}$, respectively.

\section{METHODS}

The sample consists of a $25 \mathrm{~nm}$ GZO layer on a $160 \mathrm{~nm}$ $\mathrm{ZnO}$ layer, which are deposited with molecular beam epitaxy on a $3 \mu \mathrm{m} \mathrm{GaN}$ template. The GaN template is grown on a $500 \mu \mathrm{m}$ sapphire $\left(\mathrm{Al}_{2} \mathrm{O}_{3}\right)$ substrate with metalorganic chemical vapor deposition at $1060^{\circ} \mathrm{C}$ after a buffer layer of $\mathrm{GaN}$ is formed at $530^{\circ} \mathrm{C}$. The $\mathrm{ZnO}$ and $\mathrm{GZO}$ layers are grown at 570 and $250{ }^{\circ} \mathrm{C}$, respectively, after a thin $\mathrm{ZnO}$ buffer layer is deposited at $300{ }^{\circ} \mathrm{C}$ on the $\mathrm{GaN}$ template. The $\mathrm{Ga}$ effusion cell temperature for growing GZO is set at $900{ }^{\circ} \mathrm{C}$. The $\mathrm{Zn}$ effusion cell temperatures for depositing $\mathrm{ZnO}$ and $\mathrm{GZO}$ are 290 and $315^{\circ} \mathrm{C}$, respectively. The RF plasma power is $350 \mathrm{~W}$ and the $\mathrm{O}_{2}$ flow rate is $1 \mathrm{sccm}$ during $\mathrm{ZnO}$ and $\mathrm{GZO}$ growths. Based on the $\mathrm{x}$-ray diffraction measurements, the full widths at half maximum (FWHMs) of the rocking curves of $\mathrm{GaN}$ and $\mathrm{ZnO}$ are 214 and 196 arcsec, respectively.

Hall-effect measurements were performed at room temperature with a Lake Shore 7507 automated apparatus. Ohmic indium contacts were applied on the corners of $10 \mathrm{~mm} \times$ $10 \mathrm{~mm}$ samples and the data were analyzed by the van der Pauw method. Carrier concentration was determined by $n=$ $1 / e R_{H}$, where $R_{H}$ is the Hall coefficient, and mobility by $\mu=$ $\sigma R_{H}$, where $\sigma$ is the conductivity. No Hall-factor corrections were necessary because the carriers are degenerate. Further details on Hall-effect measurements and theory can be found in Ref. [26].

Samples were prepared for transmission electron microscopy (TEM) investigations by standard preparation methods including mechanical grinding and polishing using an Allied Multiprep system. For final thinning, ion beam milling was performed with a Gatan PIPS II using gradually decreasing angles and voltages of the Ar beam. This resulted in a minimum sample thickness of approximately $50 \mathrm{~nm}$, as determined by the $t / \lambda$ method with EELS. The experiments were performed in an FEI Titan G2 60-300 kV TEM, equipped with a monochromator and probe corrector. To avoid carbon contamination, the samples were cleaned using a Fischione
Model 1020 plasma cleaner prior to the TEM experiments. Scanning transmission electron microscopy (STEM) imaging and energy-dispersive x-ray spectroscopy (EDS) were performed with the highest acceleration voltage of $300 \mathrm{kV}$ with a convergence angle of $30 \mathrm{mrad}$, while EDS was performed using the FEI Super-X EDS system and analyzed with Bruker Esprit software. For cooling experiments, a Gatan 636 Liquid Nitrogen cryo holder was used.

Electron energy-loss spectroscopy (EELS) was measured with a Gatan $965 \mathrm{GIF}$, and was performed at $60 \mathrm{kV}$ in order to reduce effects from Cherenkov radiation [27,28]. With a STEM convergence angle of $30 \mathrm{mrad}$ and an EELS collection angle of $27 \mathrm{mrad}$, most of the reciprocal space is in the central spot and collected in the GIF; hence the acquired spectra may contain contributions from transitions with a large range of momentum transfers. With the monochromator enabled and a dispersion of $0.01 \mathrm{eV} / \mathrm{ch}$, the system resolution was estimated to $0.13 \mathrm{eV}$ as measured by the FWHM of the zero-loss peak (ZLP). EELS was measured in spectrum image mode to collect data with two spatial dimensions and one energy loss dimension. Immediate dark correction was applied to the spectra after the acquisitions, and the ZLP maximum was used for calibrating the energy loss in each spectrum. A simultaneously acquired STEM ADF image was used for determining the position of the sample surface within the corresponding data set. Noise was reduced by applying principal component analysis (PCA) [29] with 24 components.

In order to assess the contribution from transitions with momentum transfer, simulations of EELS were performed using a simplified band structure consisting of a single valence band and a single conduction band. The the crystal was simplified to a cubic unit with sides $a=3.25 \AA$. The doubledifferentiated scattering cross section was calculated for all possible transitions in the first Brillouin zone, considering all transitions corresponding to excitations into available states in the conduction band [30-32]. As information of both momentum and energy transfer were preserved, a simulation of EELS could be obtained by summing the calculated transitions over the entire Brillouin zone. Furthermore, the contributions of nonoptical transitions were separated from optically allowed transitions by a cutoff value of $0.07 \mathrm{mrad}$.

\section{RESULTS AND DISCUSSIONS}

\section{A. Structure and optical characterization}

Figure 1(a) shows a cross-sectional high angle annular dark field (HAADF) STEM image of the ZnO/GZO films. In this image mode, the contrast is dominated by mass contrast, and the small difference between $\mathrm{ZnO}$ and $\mathrm{GZO}$ is ascribed to the similarity of $\mathrm{Ga}$ and $\mathrm{Zn}$ masses. The GZO film has a certain surface roughness and the film thickness in the growth direction is found to be approximately $30 \mathrm{~nm}$, slightly more than the nominal thickness of $25 \mathrm{~nm}$. Both $\mathrm{ZnO}$ and GZO films show epitaxial growth with few structural defects. Figure 1(b) shows the simultaneously acquired annular dark field (ADF) STEM image, where the GZO shows more lattice strain than $\mathrm{ZnO}$, and the interface become more visible than in HAADF. Figures 1(c) and 1(d) show EDS maps of Zn and $\mathrm{Ga} K$ edges in the same area. There is an increased $\mathrm{Ga}$ 

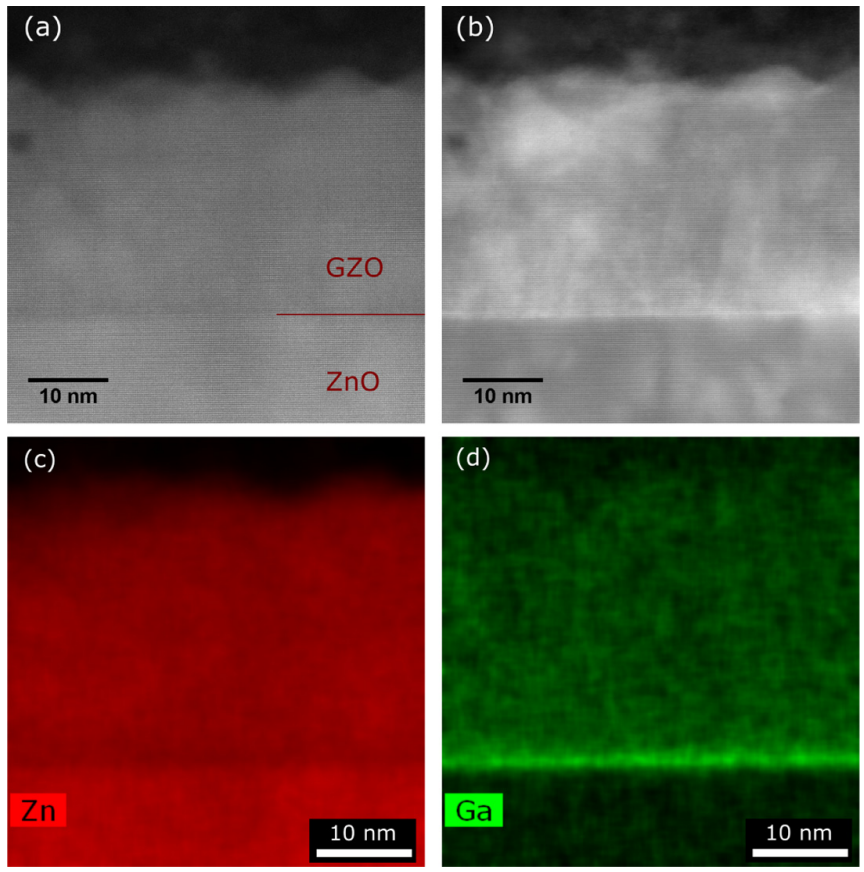

FIG. 1. Cross section view of the $\mathrm{ZnO} / \mathrm{GZO}$ interface, by HAADF (a) and ADF (b) STEM imaging, and STEM-EDS Zn (c) and $\mathrm{Ga}(\mathrm{d})$ maps.

signal by the interface which could be related to a channeling effect [33], whereas the rest of the film shows a uniform Ga content of $6.1 \pm 0.2$ at. \% relative to $\mathrm{Zn} \mathrm{(3} \mathrm{at.} \mathrm{\%} \mathrm{Ga} \mathrm{in} \mathrm{the}$ film).

Low-loss EEL spectra measured in the $\mathrm{ZnO}$ and GZO films are shown in Fig. 2, including the large ZLP which was used for energy calibration. When comparing the $\mathrm{ZnO}$ and GZO spectra, two main differences can be observed, as indicated by the arrows. First, the edge around $3-4 \mathrm{eV}$ has a different shape, appearing with a sharp onset in $\mathrm{ZnO}$ but more gradual

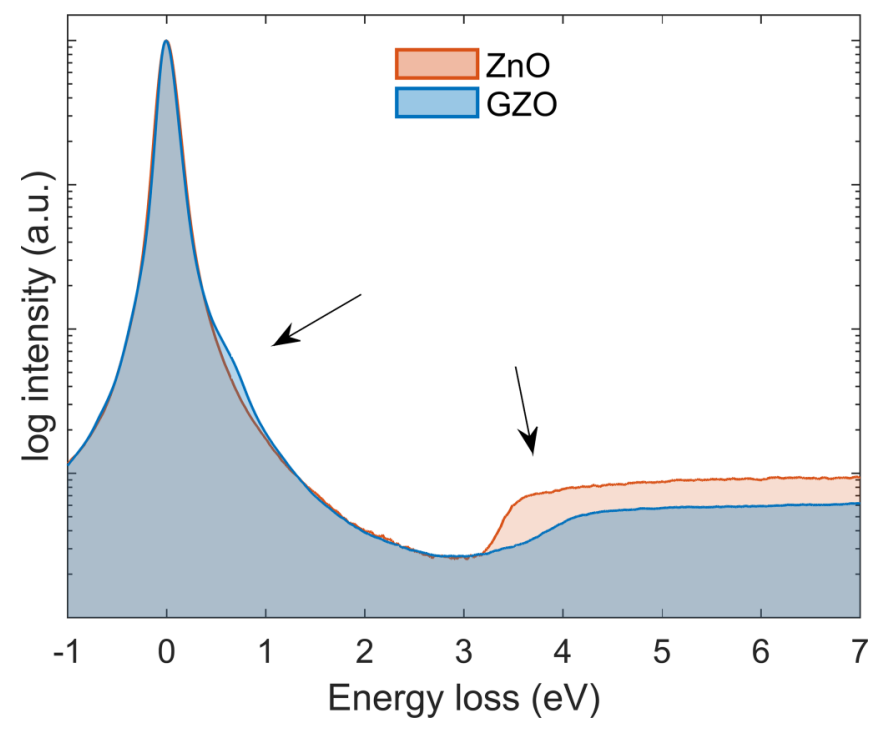

FIG. 2. EELS of the GZO and $\mathrm{ZnO}$ films. The arrows indicate the features discussed in the text.

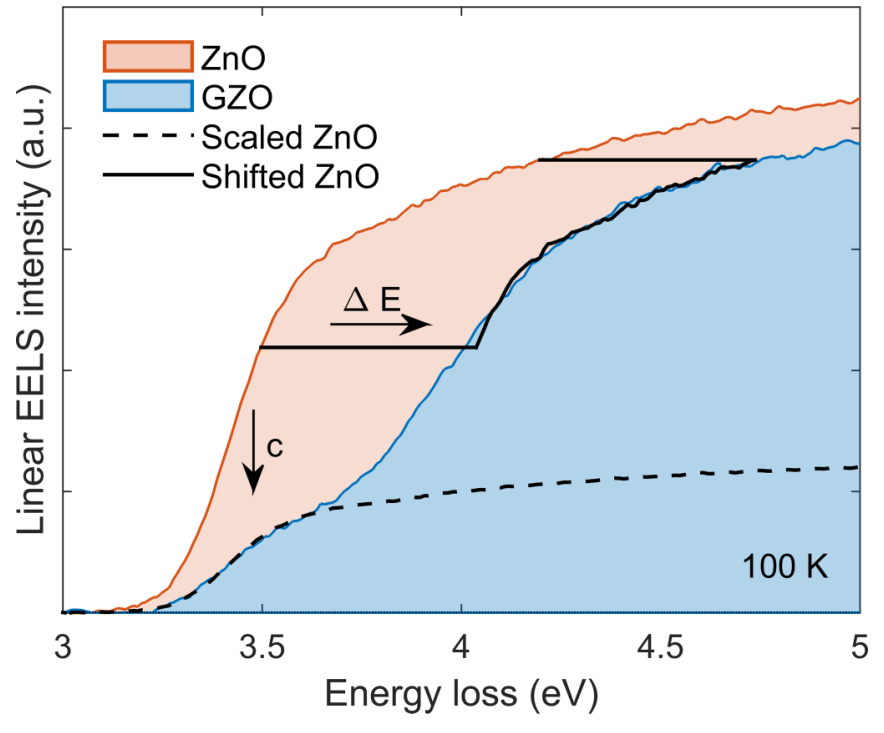

FIG. 3. Energy-loss edge of $\mathrm{ZnO}$ and GZO measured at $100 \mathrm{~K}$ after background subtraction, showing $\mathrm{ZnO}$ contributions in the GZO edge. The GZO spectrum displays a distinct shoulder on the low energy side before the main edge. The size of this shoulder varies across the GZO film, being more prominent near the $\mathrm{ZnO}$ interface and weaker towards the sample surface.

and less distinct in GZO. Secondly, below $1 \mathrm{eV}$ a broad intensity appears as a shoulder to the ZLP in GZO, which is not present in $\mathrm{ZnO}$. Both effects will be described in detail in the following.

\section{B. Band gap analysis}

A closeup of the $\mathrm{ZnO}$ and GZO band gap energy-loss edges is provided in Fig. 3. These were measured at $100 \mathrm{~K}$ in order to sharpen the significant features, whereas the same trends are also seen in room temperature measurements. The background from the ZLP was subtracted from the spectra by fitting a power-law function in an energy range right below the edge [34]. In EELS, the optical band gap is related to the onset of energy-loss, similar to an onset of optical absorption in optical spectroscopy methods. The optical gap $E_{\mathrm{g}}$ of a direct gap semiconductor can be evaluated by [35]

$$
I(E)=c\left(E-E_{\mathrm{g}}\right)^{1 / 2},
$$

provided the structure can be simplified by a parabolic valence band and a parabolic conduction band. A curve fit of this function to the spectrum measured in $\mathrm{ZnO}$ yields an energy-loss onset of $3.35 \mathrm{eV}$ at $100 \mathrm{~K}$, and $3.32 \mathrm{eV}$ at room temperature (RT). The standard deviation is below $0.01 \mathrm{eV}$ based on 19 measurements in the $\mathrm{ZnO}$ layer, and we therefore consider this variation to be significant. This is also consistent with the optical band gap of $\mathrm{ZnO}$ and its temperature dependence as previously found by EELS [36], as well as by other conventional band gap methods [37].

In samples with high enough electron concentrations, such as ours, the Fermi level moves into the conduction band and thus states below this level are filled and not available for optical transitions from the valence band. This phenomenon leads to an increase in the optical band gap, the so-called 


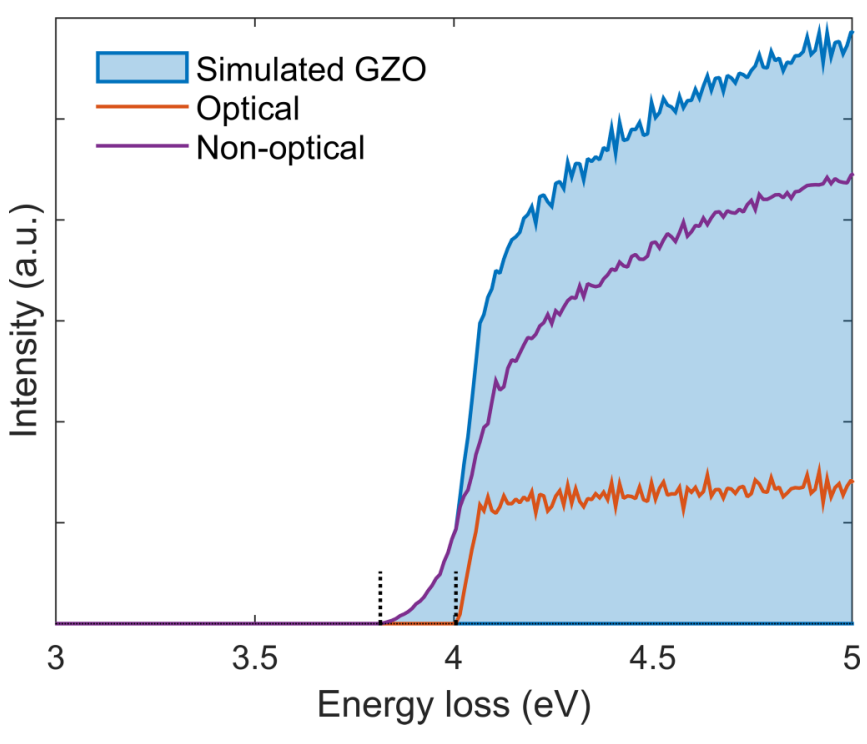

FIG. 4. Simulated Burstein-Moss shift in GZO with Fermi level $0.5 \mathrm{eV}$ above the CBM. Separation of contributions from transitions without momentum transfer (optical) and with (nonoptical) momentum transfer yield different onsets.

Burstein-Moss shift. Hence a shift in the onset of energy loss should be expected in GZO. Considering now the measured GZO spectrum in Fig. 3, the main edge appears shifted in GZO compared to $\mathrm{ZnO}$, as is marked with $\Delta E$. The shift is approximately $0.5 \mathrm{eV}$, and we interpret this as the BursteinMoss shift in GZO.

However, in contrast to the expected Burstein-Moss shift, the GZO edge in Fig. 3 shows that the onset is located around the same energy as $\mathrm{ZnO}$. The edge has a different shape than in $\mathrm{ZnO}$, thereby showing intensity below the expected onset. As we are here employing electrons for probing the material, an important aspect to consider may then be the transfer of momentum during an interaction. With a heavily doped film, the Fermi level is placed above the conduction band minimum (CBM), and a transition from the valence band maximum to the first available state should therefore involve a small momentum transfer. In EELS, in the absence of direct band gap transitions, the spectra from transitions with momentum transfer are evaluated by [35]

$$
I(E)=c\left(E-E_{\mathrm{g}}\right)^{3 / 2} .
$$

Hence transitions with momentum transfer appear with a less steep edge than direct, forward-scattered transitions, which may partly resemble the shape of the GZO edge in Fig. 3.

To consider momentum transfer as an effect in the GZO EELS results, simulations of the resulting spectrum with and without momentum transfer were performed. With a simple band structure with a band gap of $3.32 \mathrm{eV}$, the Fermi level was placed well into the conduction band, and all transitions between occupied valence states into available states above the conduction band edge were calculated. Momentum transfer was included in the calculation, and the contributions from nonoptical transitions could be separated from the direct, optical transitions by a cutoff value of $0.07 \mathrm{mrad}$. A resulting spectrum from these simulations is shown in Fig. 4. The onset

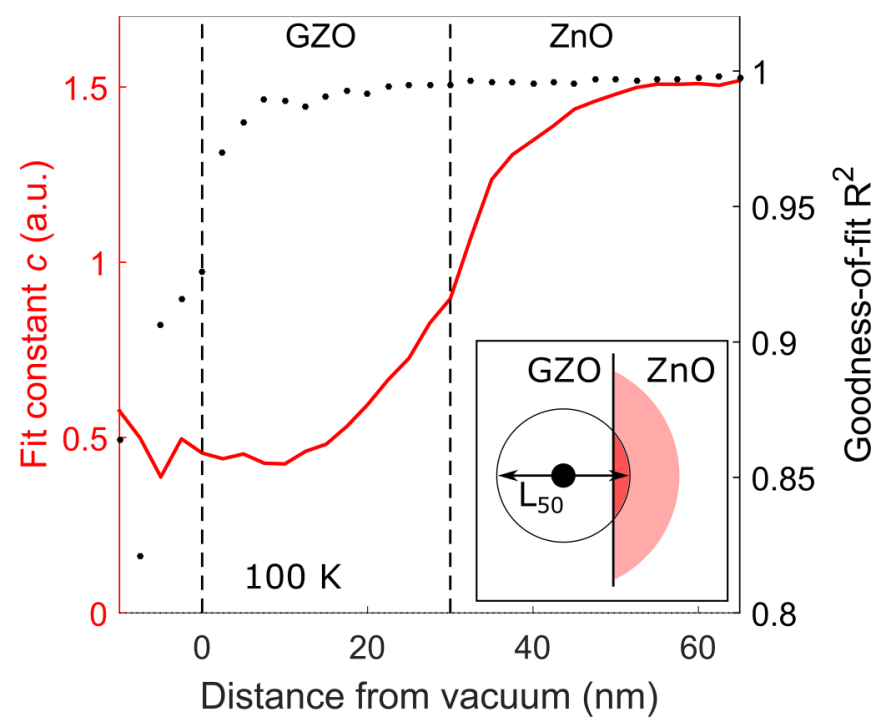

FIG. 5. Line profile of the $\mathrm{ZnO}$ onset fitting constant $c$ (fully drawn red line), and the $\mathrm{R}^{2}$ value of the fitting (dotted black). The fitting constant demonstrates an appreciable contribution to the signal from $\mathrm{ZnO}$ far into the GZO film, demonstrating the delocalization of the signal in line with theory and previous observations. The inset illustrates inelastic delocalization, where a $\mathrm{ZnO}$ signal is measured in the GZO.

of the direct, optical transitions is at $4.0 \mathrm{eV}$, as determined by the position of the Fermi level. Moreover, the simulation shows that momentum transfer has a nonzero contribution below the optical onset, with the onset of nonoptical transitions of approximately $3.8 \mathrm{eV}$. However, this difference of $0.2 \mathrm{eV}$ indicates that momentum transfer cannot account for the full energy range of the intensity observed below the edge and, furthermore, these contribute with a relatively low intensity in the edge below the onset of direct transitions.

The GZO spectrum in Fig. 3 shows the onset has a feature of the same shape and energy as the $\mathrm{ZnO}$ edge, but with lower intensity. By scaling the $\mathrm{ZnO}$ edge to this feature, there is a clear resemblance between the $\mathrm{ZnO}$ and GZO edge, as illustrated by scaling $c$ in the figure. Furthermore, spatially resolved STEM-EELS measurements show that the intensity of this feature changes across the GZO, with a gradual increase towards $\mathrm{ZnO}$. Hence this is interpreted as a $\mathrm{ZnO}$ signal, which is measured in GZO due to inelastic delocalization [21]. In short, due to the extended electric field from the TEM electron, interactions can take place at a certain distance from the position of the beam. The interaction volume depends on the amount of energy which is transferred, and hence the delocalization depends on the energy loss in question. Inelastic delocalization is typically described by the parameter $L_{50}$, the diameter contributing with $50 \%$ of the signal, and, for energy losses corresponding to the $\mathrm{ZnO}$ band gap, previous experiments show this is on a 7-8 nm range [25], consistent with previously proposed models [21]. Hence, even at some distance from the $\mathrm{ZnO}$ film, a certain portion of the acquired signal will still originate from $\mathrm{ZnO}$, as illustrated in the inset of Fig. 5. Thus a $\mathrm{ZnO}$ contribution to the signal is expected on the length scales studied here, which should be most 
pronounced by the interface, and decrease with distance from the $\mathrm{ZnO}$.

If the intensity is indeed the delocalized $\mathrm{ZnO}$ signal, it can be analyzed in the same manner as the $\mathrm{ZnO}$ edge. By fixing the onset to the measured onset of $\mathrm{ZnO}$, the measured amount of $\mathrm{ZnO}$ edge was quantified by the fit constant $c$ when fitting Eq. (1). Figure 5 presents the resulting line profile of the $\mathrm{ZnO}$ edge fitting across the GZO, shown as a function of distance into the sample. The scaling $c$ of the fit, corresponding to the intensity of a $\mathrm{ZnO}$ contribution, is here seen to gradually decrease away from the $\mathrm{ZnO}$, which is in good agreement with inelastic delocalization. Furthermore, the corresponding goodness-of-fit parameter $\mathrm{R}^{2}$ is also shown in the figure, indicating a high fit quality. Hence the edge measured in the GZO is interpreted as a combination of delocalized signal from $\mathrm{ZnO}$, and a Burstein-Moss shift in GZO.

Due to the $\mathrm{ZnO}$ contributions, an accurate assessment of the Burstein-Moss shift in GZO is difficult. However, assuming that the shape of the edge is the same in $\mathrm{ZnO}$ and $\mathrm{GZO}$, the energy shift could be found by fitting the shape of the $\mathrm{ZnO}$ feature to the GZO, as shown with $\Delta E$ in Fig. 3. To quantify $\Delta E$, the $\mathrm{ZnO}$ spectrum range was here extracted and compared to the GZO by curve fitting. This procedure was applied to a line profile across the GZO measured at RT, and the result is shown in Fig. 6(a). At the edges of the GZO, the method is seen to become less accurate due to a less pronounced edge with more noise, as verified by manual inspection of the spectra.

Additionally, the resulting mapping of the energy shift $\Delta E$ across the GZO and $\mathrm{ZnO}$ interface is shown in Fig. 6(b). Here, the energy shift is assumed to be approximately uniform across the GZO film, in accordance with the EDS results, and the observed weak variation along the GZO may be ascribed to noise introduced by the fitting procedure. Furthermore, this shows that the average energy shift is $\Delta E=0.52 \mathrm{eV}$ with a standard deviation of $0.08 \mathrm{eV}$.

To confirm that the energy shift found by EELS corresponds to the change in band gap due to Ga doping, we have calculated the corresponding charge carrier concentration $n$. This can be compared to a previous Hall measurement, resulting in the estimate $n=7 \times 10^{20} \mathrm{~cm}^{-3}$. In a rigid band approximation, the Burstein-Moss shift can be described by [38]

$$
\Delta E_{\mathrm{BM}}=\frac{\hbar^{2}}{2 m_{\mathrm{cv}}^{*}}\left(3 \pi^{2} n\right)^{2 / 3},
$$

where $1 / m_{\mathrm{cv}}^{*}=1 / m_{\mathrm{c}}^{*}+1 / m_{\mathrm{v}}^{*}$ is the reduced effective mass. In a parabolic approximation, the effective masses $m_{c}^{*}=$ $0.28 m_{0}$ [39] and $m_{v}^{*}=1.37 m_{0}$ [40] may describe the band structure. However, as we here observe a relatively large shift in energy, the Fermi level is moved far into the conduction band, possibly outside the range where a parabolic description of the conduction band dispersion is valid. A modification can be made by including a term for nonparabolic effective mass, which can be expressed by $[41,42]$

$$
m_{\mathrm{c}, \mathrm{NP}}^{*}=m_{c}^{*} \sqrt{1+2 C \frac{\hbar^{2}}{m_{c}^{*}}\left(3 \pi^{2} n\right)^{2 / 3}} .
$$
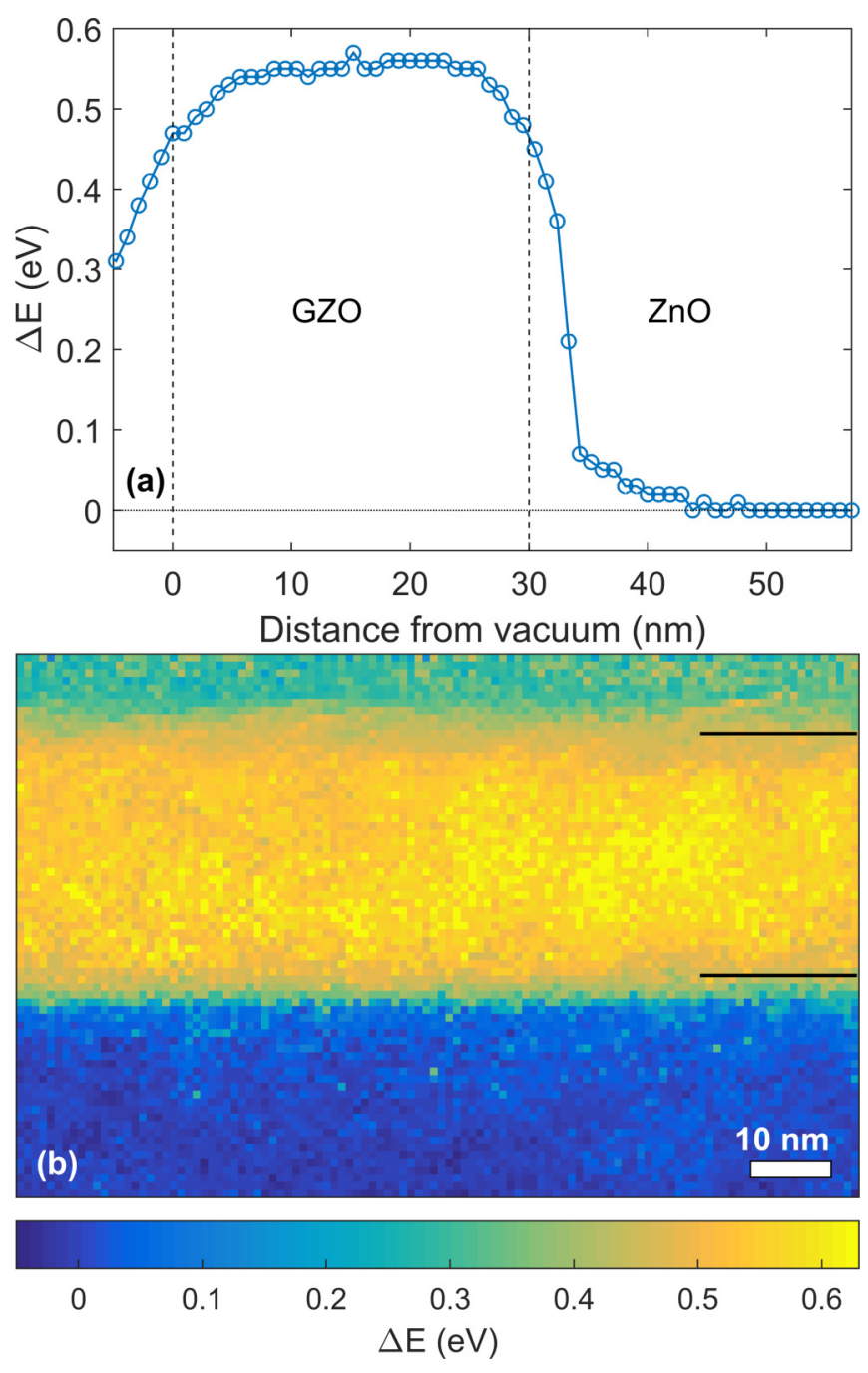

FIG. 6. Line profile (a) and mapping (b) of the Burstein-Moss shift across the GZO film.

$C$ represents the inverse of the optical band gap [43], which here corresponds to the RT $\mathrm{ZnO}$ onset at $3.32 \mathrm{eV}$.

Furthermore, band gap renormalization will be a significant factor in these calculations. This effect appears due to hybridization between a defect level and the conduction band, and leads to band gap narrowing (BGN) $[44,45]$. If this effect is taken into account, the total energy shift we observe may be expressed as

$$
\Delta E=\Delta E_{\mathrm{BM}, \mathrm{NP}}-\Delta E_{\mathrm{BGN}} .
$$

A model for band gap renormalization previously applied to $\mathrm{ZnO}$ is [45]

$$
\Delta E_{\mathrm{BGN}}=B n^{1 / 3}\left[1-\left(\frac{n_{c}}{n}\right)^{1 / 3}\right],
$$

with the parameters for the critical charge carrier density $n_{c}=3.87 \times 10^{18} \mathrm{~cm}^{-3}$ and the constant $B=5 \times 10^{-8} \mathrm{~m}^{-3}$. The corresponding carrier concentration calculated from the observed energy shift $\Delta E$ is shown in Fig. 7: using the observed energy shift of $\Delta E=0.52 \pm 0.08 \mathrm{eV}$, the resulting charge carrier concentration extracted from EELS is found 


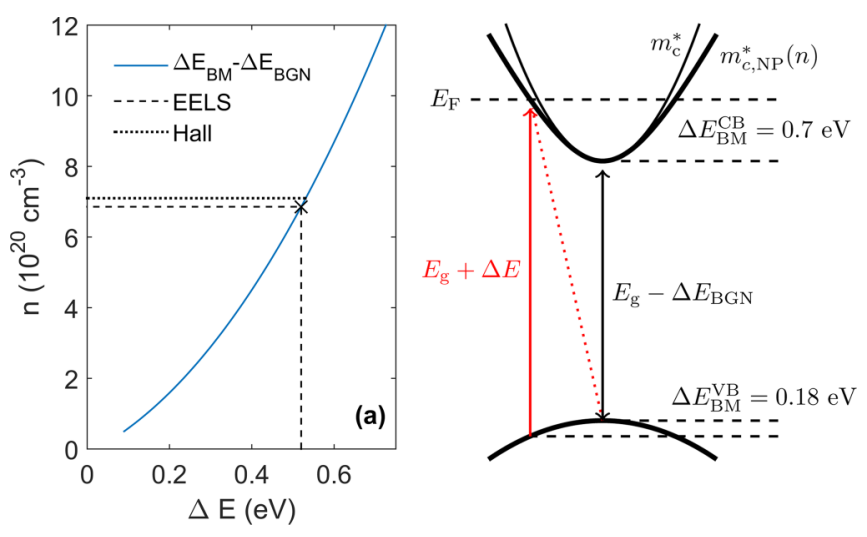

FIG. 7. (a) Relation between charge carriers $(n)$ and band gap shift $(\Delta E)$. The band gap shift is explained by a combination of a nonparabolic Burstein-Moss shift ( $\left.\Delta E_{\mathrm{BM}}\right)$ and band gap narrowing $\left(\Delta E_{\mathrm{BGN}}\right)$. The band gap shift from EELS yields a very close correspondence in $n$ with Hall measurements. (b) The renormalized band structure showing the effect of the Burstein-Moss shift in the valence and conduction bands. The red lines show the measured transitions, without (whole line) and with (dotted line) momentum transfer.

to be $6.9 \pm 1.7 \times 10^{20} \mathrm{~cm}^{-3}$. This is in very close correspondence with the result of $7 \times 10^{20} \mathrm{~cm}^{-3}$ obtained by Hall measurements.

The measured energy shift can be separated into a BM shift of $0.88 \mathrm{eV}$ and a BGN of $0.36 \mathrm{eV}$. The band structure model is illustrated in Fig. 7(b), where the valence and conduction bands were separated by $E_{\mathrm{g}}=3.32 \mathrm{eV}$ and then renormalized by $\Delta E_{\mathrm{BGN}}$. The nonparabolic effective mass of the conduction band becomes $m_{\mathrm{c}, \mathrm{NP}}^{*}(n)=0.42 m_{0}$. As can be seen from Fig. 7(b) and the observed BM shift, it becomes clear that the conduction band deviates from the parabolic description. Furthermore, the BM shift can be split into observed energy shifts in both valence and conduction bands, as the onset of direct transitions now occurs at $k>0$. With the effective masses found above, the energy shifts in each band can be found. Here we see that most of the BM shift is within the conduction band; however, a $0.18 \mathrm{eV}$ shift occurs within the valence band. This energy difference corresponds to the difference between optical and nonoptical transitions (marked in the figure with red line and red dotted line, respectively), and is also in very close agreement with the simulated spectrum in Fig. 4.

In summary, the GZO edge showed a shift of $0.52 \mathrm{eV}$, which could be described by a BM shift of $0.88 \mathrm{eV}$ in combination with BGN of $0.36 \mathrm{eV}$. The effect of momentum transfer has also been discussed, showing very little contribution to the spectrum, whereas delocalized signal from $\mathrm{ZnO}$ was observed below the onset of direct transitions. Furthermore, the energy shift has been mapped with high spatial resolution across the $\sim 25 \mathrm{~nm}$ film and is in good agreement with the EDS results.

\section{Plasmon analysis}

The second feature observed in Fig. 2 is a broad shoulder occurring in GZO located below $1 \mathrm{eV}$. A closeup of this feature is provided in Fig. 8(b), where the difference to a scaled $\mathrm{ZnO}$-measured spectrum is highlighted. Furthermore, Fig. 8(a) shows the position the spectra were extracted, and here we also observe a variation in the peak energy. To confirm that this is a real feature, deconvolution using the Richardson-Lucy algorithm with a Lorentzian-Gaussian kernel was applied to the data with 15 iterations. The spectra after deconvolution are shown in Fig. 8(c), where the feature is clearly enhanced while no other artifacts appear in the GZO spectra.

As the peak occurs below the Kimoto limit [46], we found that an accurate determination of the position is mainly limited by the choice of method for background subtraction. Considering one GZO spectrum, different choices of background models and fitting methods lead to a variation of the peak position in the range $0.80-0.90 \mathrm{eV}$. We have found that a reliable method was to use the data without deconvolution, subtract a scaled $\mathrm{ZnO}$ spectrum, and extract the peak position by fitting a two-Gaussian model. This is shown in Fig. 9(a), and the peak could then be interpreted in terms of peak energy and intensity. By this method, we find that the peak in GZO is located at $0.82 \mathrm{eV}$.

The peak we observe in the GZO is interpreted as a plasma resonance. It is well known that the sample may interact with the electromagnetic field of the TEM electrons by setting up a collective oscillation of the free electrons (plasmons). The frequency of this oscillation depends on the density of free charges, where the Drude model gives the plasmon energy of a free electron gas as [32]

$$
E_{\mathrm{p}}=\hbar \omega_{\mathrm{p}}=\hbar \sqrt{\frac{n e^{2}}{\epsilon_{0} m_{0}}} .
$$

Here $n$ is the density of free charges, $e$ is the electron charge, $\epsilon_{0}$ is the permittivity of free space, and $m_{0}$ is the electron mass. In undoped samples, EELS is often used for studying the volume plasmon generated by the valence band. $n$ is then the density of valence electrons, and in $\mathrm{ZnO}$ the plasmon occurs as a broad peak centered at $18.9 \mathrm{eV}$ [47]. However, in heavily doped samples with a significant amount of charge carriers in the conduction band, the conduction band can set up its own plasmon oscillation [48-51]. The energy of this conduction band plasmon can be described according to the above equation, with $n$ being the density of electrons in the conduction band, hence the charge carrier concentration.

Using the charge carrier concentration $n$ extracted from the Burstein-Moss shift found with EELS with Eq. (7), we calculate a conduction band plasmon energy of $E_{\mathrm{p}}=0.98 \mathrm{eV}$. However, Eq. (7) is based on a system of free electrons, which may not be accurate for the current situation. Modifications of the model can be made by replacing $m_{0}$ with $m_{\mathrm{c}, \mathrm{NP}}^{*}$ and $\epsilon_{0}$ with $\epsilon_{r} \epsilon_{0}$, thus considering electrons in the GZO conduction band and the dielectric response of the material. At these energy losses, the dielectric constant of $\mathrm{ZnO}$ is approximately $\epsilon_{r}=3.71$ [52], and one obtains $E_{\mathrm{p}}=0.78 \mathrm{eV}$. This is in very close agreement with the observed peak and hence we conclude that the observed peak is the plasma resonance from the conduction band.

Furthermore, the conduction band electrons participate in the oscillation with an effective mass which varies with the electron energy (position in the conduction band). A better estimate of the plasmon may therefore be found by calculating the average effective mass of all the participating electrons. 

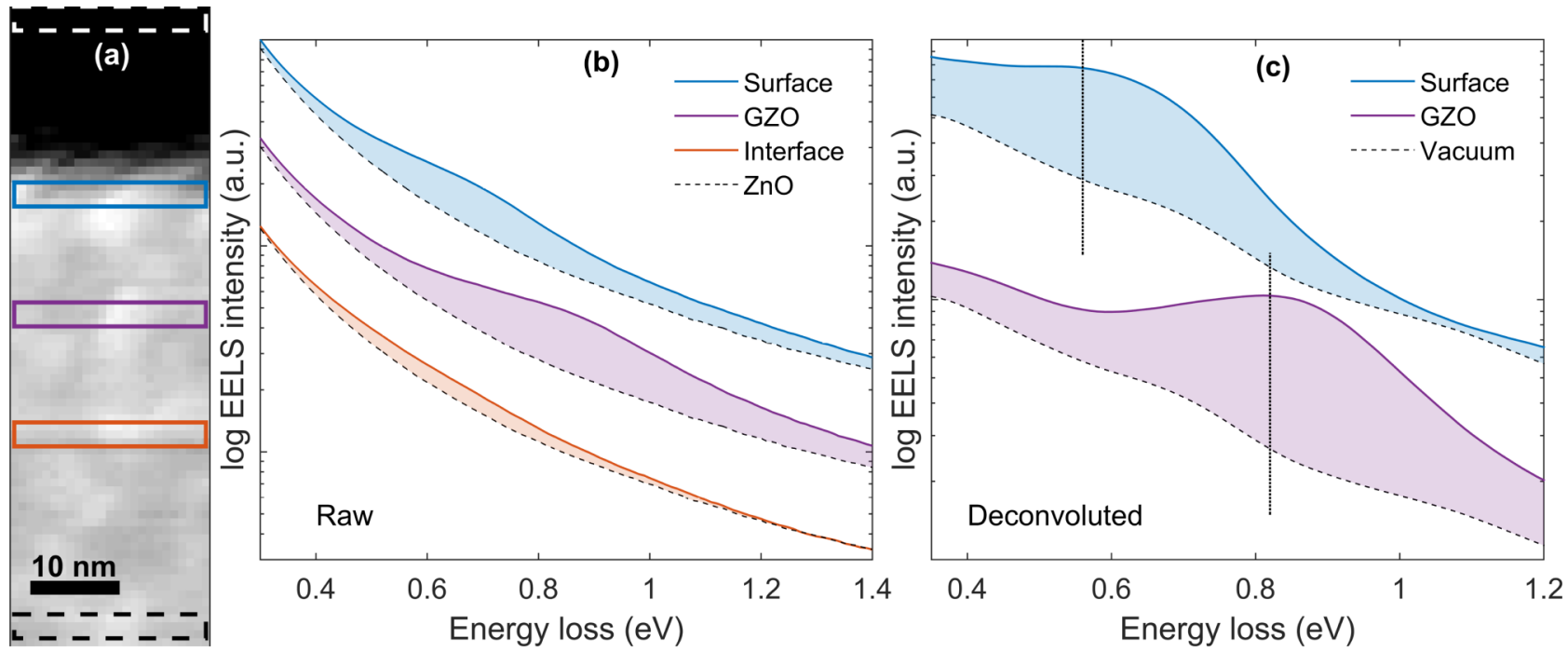

FIG. 8. ADF image (a) and spectra before (b) and after (c) deconvolution (log scale), showing spatial variation of plasmon energy across the GZO.

Considering a range of charge carriers between zero and the measured $n$, the corresponding range of nonparabolic effective masses can be calculated from Eq. (4), thus yielding the average nonparabolic effective mass of $0.37 m_{0}$. Also, due to the presence of the plasmon, the dielectric function of GZO may be different than for $\mathrm{ZnO}$. Therefore, we can instead calculate the GZO dielectric constant at the observed plasmon energy, based on the observed plasmon energy, the charge
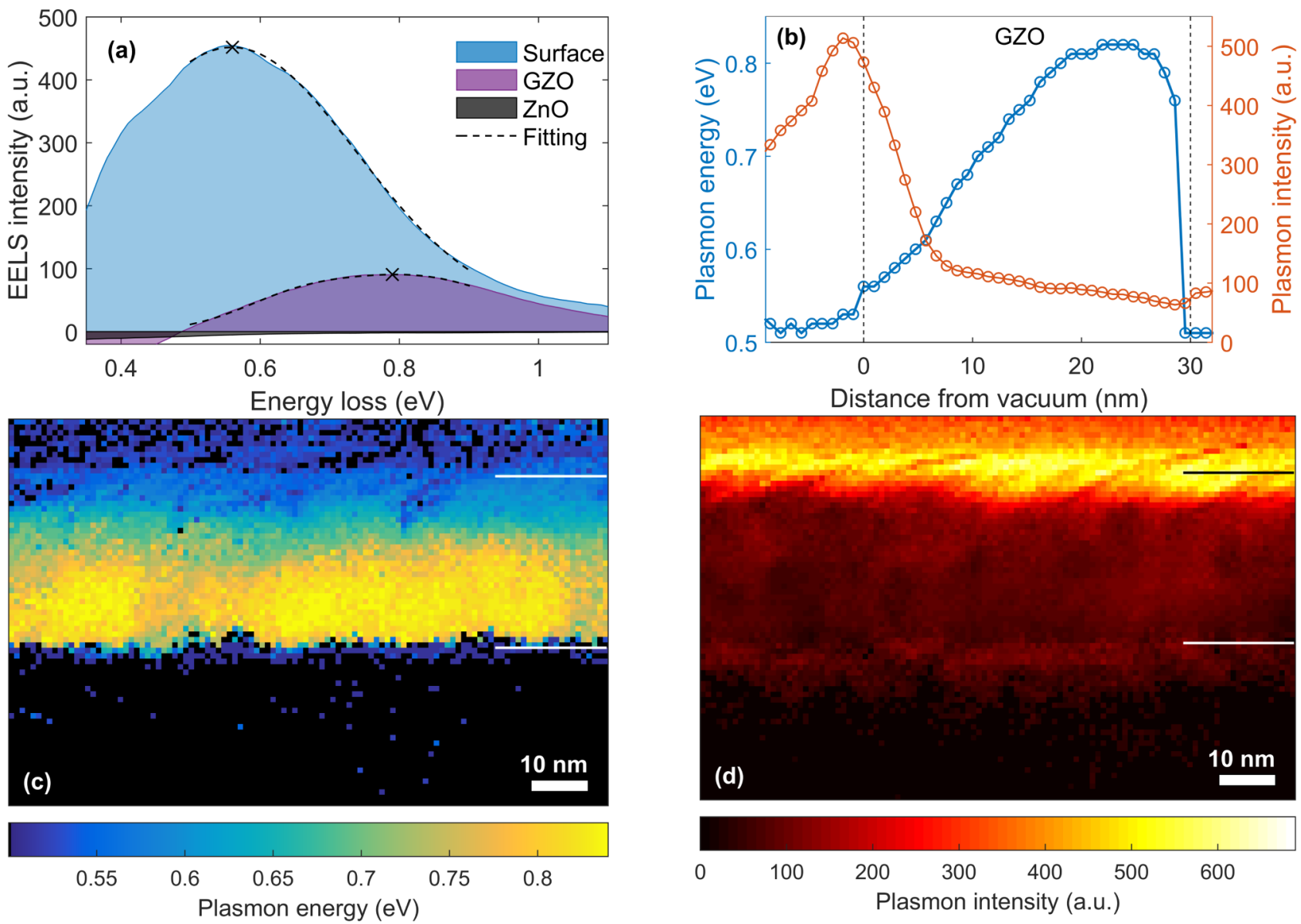

FIG. 9. (a) Plasmon peak analysis without deconvolution, where a scaled $\mathrm{ZnO}$ spectrum was used for background subtraction. (b) Line profile of the plasmon energy and intensity across GZO, and mapping of the plasmon energy (c) and intensity (d). An energy shift towards the surface is observed, which is interpreted as the corresponding surface plasmon. 
carrier concentration from EELS, and the average effective mass. This results in $\epsilon_{r}=3.8 \pm 0.9$. Due to the relatively large error found for $n$, the difference from the $\mathrm{ZnO}$ value of $\epsilon_{r}=3.71$ is not found to be significant.

As seen in Fig. 8(b), there is a spatial variation of the plasmon energy of more than $0.2 \mathrm{eV}$. A spatially resolved quantification of the peak was performed with the method described above, shown in Fig. 9(a). Figure 9(b) shows the line profile across the GZO film, and a complete mapping of peak intensity and energy is shown in Figs. 9(c) and 9(d). The plasmon energy is here seen to reach a maximum in the bottom half towards the $\mathrm{ZnO}$ interface, which corresponds to the bulk plasmon energy discussed above.

Interestingly, Fig. 8 shows that the plasmon peak clearly shifts across the film, with decreasing energy and increasing intensity towards the surface. This variation does not correlate with any change in Ga content or charge carrier concentration, according to the previous EDS results and Burstein-Moss shift. The surface region displays a lower plasmon energy than the interior of the film, and this is interpreted as the excitation of a surface plasmon mode. For a volume plasmon of energy $E_{\mathrm{p}}$, the free electron model results in a corresponding surface plasmon located at [32]

$$
E_{\mathrm{sp}}=E_{\mathrm{p}} / \sqrt{2} \text {. }
$$

For the bulk plasmon energy observed here, a surface plasmon energy of $E_{\mathrm{sp}}=0.82 \mathrm{eV} / \sqrt{2}=0.58 \mathrm{eV}$ should be expected. The EELS line profile in Fig. 9(b) shows the plasmon at the surface (distance from vacuum $=0 \mathrm{~nm}$ ) has an energy $0.56 \mathrm{eV}$. This is very close to the maximum plasmon intensity, which shows a decaying intensity on both vacuum and GZO sides attributed to inelastic delocalization. Hence, as the peak is localized at the surface with excellent correspondence in energy, we conclude that this is the surface plasmon of the GZO film.

The gradual shift in bulk plasmon energy across the GZO is here explained by delocalization of the intense surface plasmon, which may interfere with the assessment of the bulk plasmon energy. Due to the broadness of the plasmon peak, these two contributions are difficult to separate, and the combination of the two will therefore appear as a small peak shift. Furthermore, the method of extraction may also favor peak positions at lower energies, as these may be enhanced by the intense background. Thus contributions from the surface plasmon may affect the extracted energies of the bulk plasmon. There should also be a surface plasmon forming on the top and bottom sides of the TEM sample, perpendicular to the viewing direction; however, their intensities are assumed to be very small compared to the bulk plasmon. Moreover, an interface plasmon should also occur along the interface between $\mathrm{ZnO}$ and GZO. There may be indications of this in the raw spectrum in Fig. 8(b) and in the mapping in Fig. 9(c), but since these occur at low energies which are difficult to accurately extract, a clear conclusion cannot be drawn. Such interface plasmons, which would occur at energies lower than $0.4 \mathrm{eV}$, have too low energy to be trustworthily extracted here. However, the presence of interface plasmons may explain the seemingly abrupt decrease in plasmon energy at the interface seen in Fig. 9(b), as the presence of the interface plasmon may affect the background subtraction.

To summarize, high spatial resolution STEM-EELS reveals the difference between the bulk and surface effects for the conduction band plasma resonance. Here, the bulk plasmon is observed at $0.82 \mathrm{eV}$, in very good correspondence with theory, while the corresponding surface plasmon is observed at $0.56 \mathrm{eV}$.

\section{CONCLUSIONS}

In this work, we have used STEM-EELS to study the optical properties of a highly Ga-doped $\mathrm{ZnO}$ thin film of $\sim 25 \mathrm{~nm}$. Even though contributions from the adjacent $\mathrm{ZnO}$ film were observed due to inelastic delocalization, clear differences were observed between GZO and $\mathrm{ZnO}$. Exploiting the high spatial resolution of STEM-EELS, the Burstein-Moss shift has been mapped across the $25 \mathrm{~nm}$ film. When taking into account also the band gap renormalization, an overall shift of the optical band gap of $0.52 \mathrm{eV}$ was found. The corresponding charge carrier concentration was found to be $6.9 \times 10^{20} \mathrm{~cm}^{-3}$, in excellent agreement with estimates from Hall effect measurements. Furthermore, the bulk and surface plasmon of the film has been found, located at $0.82 \mathrm{eV}$ and $0.56 \mathrm{eV}$, respectively. These results are also in excellent agreement with theoretical models for the band gap and plasmons, which provide insight into the details of the band structure and dielectric properties of GZO. Hence this work shows direct mapping of the Burstein-Moss shift, which is correlated with the distribution of charge carriers, as well as enabling direct detection and mapping of the conduction band bulk and surface plasmon.

\section{ACKNOWLEDGMENTS}

Thanks to Phuong Dan Nguyen for preparing the TEM sample. This work was supported by the Faculty of Mathematics and Natural Sciences via the strategic research program FOXHOUND. The Research Council of Norway is acknowledged for the support to the Norwegian Center for Transmission Electron Microscopy (NORTEM), Project No. 197405/F50, to the Norwegian Micro- and Nano-Fabrication Facility (NorFab), Project No. 245963, and to the frontier research project FUNDAMeNT, Project No. 251131 (FriPro ToppForsk program).
[1] E. Burstein, Phys. Rev. 93, 632 (1954).

[2] T. S. Moss, Proc. Phys. Soc., London, Sect. B 67, 775 (1954).

[3] A. P. Roth, J. B. Webb, and D. F. Williams, Phys. Rev. B 25, 7836 (1982).

[4] J. Nelayah, M. Kociak, O. Stéphan, F. J. G. de Abajo, M. Tencé, L. Henrard, D. Taverna, I. Pastoriza-Santos, L. M. Liz-Marzán, and C. Colliex, Nat. Phys. 3, 348 (2007).
[5] W. Sigle, J. Nelayah, C. T. Koch, and P. A. van Aken, Opt. Lett. 34, 2150 (2009).

[6] F.-P. Schmidt, H. Ditlbacher, U. Hohenester, A. Hohenau, F. Hofer, and J. R. Krenn, Nano Lett. 12, 5780 (2012).

[7] D. C. Look and K. D. Leedy, Appl. Phys. Lett. 102, 182107 (2013).

[8] H. A. Atwater and A. Polman, Nat. Mater. 9, 205 (2010). 
[9] P. R. West, S. Ishii, G. V. Naik, N. K. Emani, V. M. Shalaev, and A. Boltasseva, Laser Photon. Rev. 4, 795 (2010).

[10] A. Boltasseva and H. A. Atwater, Science 331, 290 (2011).

[11] D. C. Look, E. R. Heller, Y.-F. Yao, and C. C. Yang, Appl. Phys. Lett. 106, 152102 (2015).

[12] C. E. Kim, P. Moon, S. Kim, J.-M. Myoung, H. W. Jang, J. Bang, and I. Yun, Thin Solid Films 518, 6304 (2010).

[13] D. C. Look, K. D. Leedy, D. H. Tomich, and B. Bayraktaroglu, Appl. Phys. Lett. 96, 062102 (2010).

[14] H. J. Ko, Y. F. Chen, S. K. Hong, H. Wenisch, T. Yao, and D. C. Look, Appl. Phys. Lett. 77, 3761 (2000).

[15] C. Moditswe, C. M. Muiva, and A. Juma, Optik-Int. J. Light Electron Opt. 127, 8317 (2016).

[16] Y. J. Park, H. N. Kim, and H. H. Shin, Appl. Surf. Sci. 255, 7532 (2009).

[17] R. Schifano, E. V. Monakhov, L. Vines, B. G. Svensson, W. Mtangi, and F. D. Auret, J. Appl. Phys. 106, 043706 (2009).

[18] P. Eyben, M. Xu, N. Duhayon, T. Clarysse, S. Callewaert, and W. Vandervorst, J. Vac. Sci. Technol. B 20, 471 (2002).

[19] D. C. Look, K. D. Leedy, G. J. Grzybowski, and B. B. Claflin, Opt. Eng. 56, 057109 (2017).

[20] K. van Benthem, R. H. French, W. Sigle, C. Elsässer, and M. Rühle, Ultramicroscopy 86, 303 (2001).

[21] R. F. Egerton, Ultramicroscopy 107, 575 (2007).

[22] M. Bosman, V. J. Keast, M. Watanabe, A. I. Maaroof, and M. B. Cortie, Nanotechnology 18, 165505 (2007).

[23] M. Bosman, L. J. Tang, J. D. Ye, S. T. Tan, Y. Zhang, and V. J. Keast, Appl. Phys. Lett. 95, 101110 (2009).

[24] L. Gu, V. B. Özdöl, W. Sigle, C. T. Koch, V. Srot, and P. A. van Aken, J. Appl. Phys. 107, 013501 (2010).

[25] W. Zhan, C. S. Granerød, V. Venkatachalapathy, K. M. H. Johansen, I. J. T. Jensen, A. Y. Kuznetsov, and $\varnothing$. Prytz, Nanotechnology 28, 105703 (2017).

[26] D. C. Look, Electrical Characterization of GaAs Materials and Devices (Wiley, New York, 1989).

[27] M. Stöger-Pollach and P. Schattschneider, Ultramicroscopy 107, 1178 (2007).

[28] R. Erni and N. D. Browning, Ultramicroscopy 108, 84 (2008).

[29] M. Bosman, M. Watanabe, D. T. L. Alexander, and V. J. Keast, Ultramicroscopy 106, 1024 (2006).

[30] S. L. Adler, Phys. Rev. 126, 413 (1962).

[31] R. H. Ritchie, Phys. Rev. 106, 874 (1957).
[32] R. F. Egerton, Electron Energy-Loss Spectroscopy in the Electron Microscope (Springer, New York, 2011).

[33] S. R. Spurgeon, Y. Du, and S. A. Chambers, Microsc. Microanal. 23, 513 (2017).

[34] C. S. Granerød, W. Zhan, and Ø. Prytz, Ultramicroscopy 184, 39 (2018).

[35] B. Rafferty and L. M. Brown, Phys. Rev. B 58, 10326 (1998).

[36] C. S. Granerød, A. Galeckas, K. M. Johansen, L. Vines, and Ø. Prytz, J. Appl. Phys. 123, 145111 (2018).

[37] B. D. Viezbicke, S. Patel, B. E. Davis, and D. P. Birnie, Phys. Status Solidi B 252, 1700 (2015).

[38] I. Hamberg, C. G. Granqvist, K.-F. Berggren, B. E. Sernelius, and L. Engström, Phys. Rev. B 30, 3240 (1984).

[39] N. N. Syrbu, I. M. Tiginyanu, V. V. Zalamai, V. V. Ursaki, and E. V. Rusu, Physica B 353, 111 (2004).

[40] W. R. L. Lambrecht, A. V. Rodina, S. Limpijumnong, B. Segall, and B. K. Meyer, Phys. Rev. B 65, 075207 (2002).

[41] T. Pisarkiewicz, K. Zakrzewska, and E. Leja, Thin Solid Films 174, 217 (1989).

[42] J. G. Lu, S. Fujita, T. Kawaharamura, H. Nishinaka, Y. Kamada, T. Ohshima, Z. Z. Ye, Y. J. Zeng, Y. Z. Zhang, L. P. Zhu, H. P. He, and B. H. Zhao, J. Appl. Phys. 101, 083705 (2007).

[43] W. M. Kim, I. H. Kim, J. H. Ko, B. Cheong, T. S. Lee, K. S. Lee, D. Kim, and T.-Y. Seong, J. Phys. D 41, 195409 (2008).

[44] G. D. Mahan, J. Appl. Phys. 51, 2634 (1980).

[45] J. Kumar and A. Srivastava, J. Appl. Phys. 115, 134904 (2014).

[46] K. Kimoto, G. Kothleitner, W. Grogger, Y. Matsui, and F. Hofer, Micron 36, 185 (2005).

[47] W. Zhan, V. Venkatachalapathy, T. Aarholt, A. Y. Kuznetsov, and Ø. Prytz, Sci. Rep. 8, 848 (2018).

[48] S. Kalusniak, S. Sadofev, and F. Henneberger, Phys. Rev. Lett. 112, 137401 (2014).

[49] M. K. Hamza, J.-M. Bluet, K. Masenelli-Varlot, B. Canut, O. Boisron, P. Melinon, and B. Masenelli, Nanoscale 7, 12030 (2015).

[50] A. Calzolari and A. Catellani, Proc. SPIE 10105, 101050G (2017).

[51] N. Nader, S. Vangala, J. R. Hendrickson, K. D. Leedy, D. C. Look, J. Guo, and J. W. Cleary, J. Appl. Phys. 118, 173106 (2015).

[52] W. L. Bond, J. Appl. Phys. 36, 1674 (1965). 